\title{
Disproval of Gravity-induced Quantum Decoherence by Micius in Space
}

By SONG Jianlan (Staff Reporter)

Once more frustrated were the ongoing efforts to bridge the two pillars of modern physics; further closer are we to the truth.

\footnotetext{
Q
} uantum mechanics and general relativity are deemed to be the two major "pillars" to support modern physics - they each are exceptionally succesful in explaining phenomena in their own realms. namely the microscopic and the macroscopic worlds, respectively. However, whenever they try to transcend the boundary to extend to the opposite, they would be frustrated with failure without any exception. For about 90 years, it has been a common dream for physicists to unify the two and establish a universal theory for the whole physical world. only to meet setbacks repeatedly.

As efforts to bridge gravity and quantum fields, many rival models of quantum gravity have arisen. but they mostly provide little foundation for experimental tests, partly because their verification demands extremely stern experimental conditions.

A team led by CAS Nember Prof. PAN Jianwei at the University of Science and Technology of China (USTC), an educational entity of CAS, took the challenge and performed an experiment aboard Micius. a satellite designed for quantum physics experiments flying at an altitude of about $500 \mathrm{~km}$ above the Earth. to test the prediction given by a model called "event formalism".

Attempting to give a coherent description of quantum fields applicable across ordinary spacetime and "exotic" spacetimes, the "event formalism" model predicted that entangled quantum pairs would decorrelate at a certain possibility when intercepting the

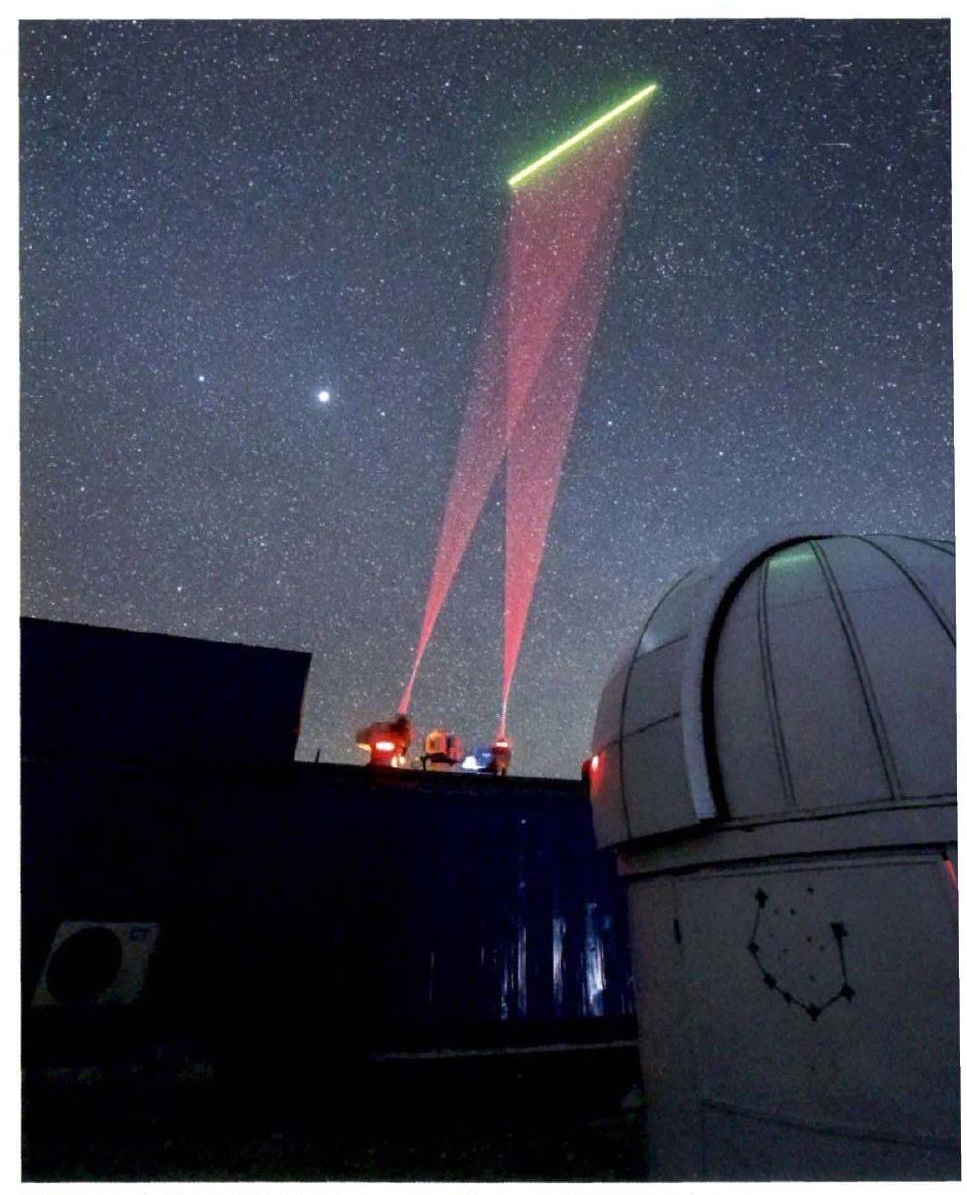

Micius, a satellite designed for quantum physics experiments flying at an altitude of about $500 \mathrm{~km}$ above the Earth, disproves the prediction given by a model of "ultimate theory" called "event formalism". (Credit: USTC) 


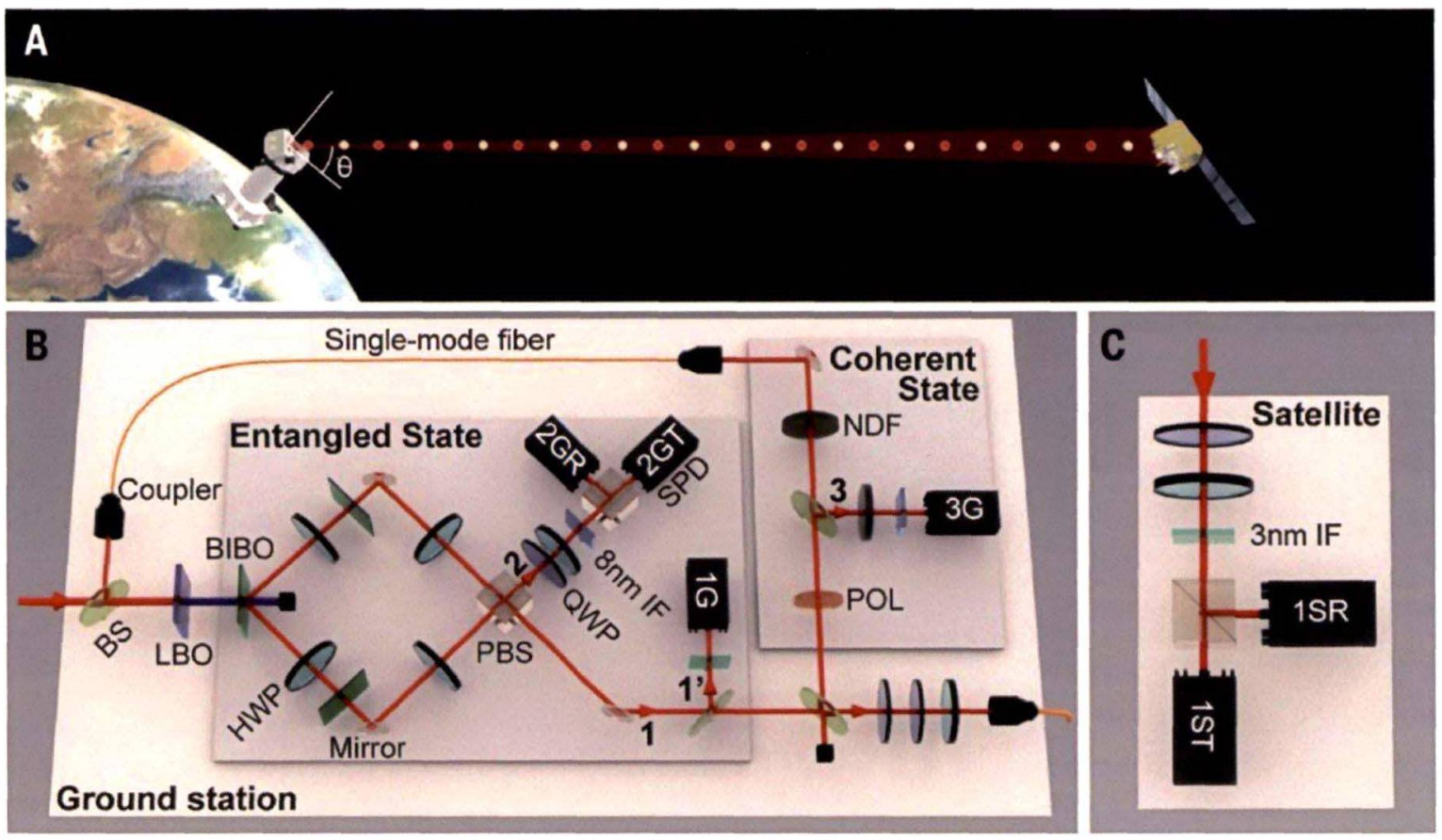

Schematics of the experimental test. The Micius experiment tests the "event formalism" model by distributing entangled photons across the space surrounding the Earth. For each pair, one photon is situated at the ground station and the other distributed to the satellite Micius. When the Earth rotates, the satellite will pass the ground station at different altitude, hence leaving the link between the twin photon of the pair cut through the space at different angles across different regions of the Earth's gravitational fields. In this process, the pair undergoes fluctuation of the potential field and would decorrelate at some possibility if the "event formalism" was right - it proves to be not. (Credit: USTC)

space surrounding the Earth, due to the fluctuation of the planet's gravitational field.

With ultra-high precision, the Micius experiment verifies that all the entangled quantum pairs distributed survived the fluctuation of the Earth's gravity. This supports the classical quantum mechanics, and disproves the prediction given by the "event formalism" model.

This work is listed the $8^{\text {th }}$ in the annual top 10 science advances of China for 2019. For a detailed story about the experiment, please refer to page 137 of lssue 3 . Vol. $3: 3$ of BCAS.

\section{Reference}

Ping Xu, Yiqiu Ma, Ji-Gang Ren, Hai-Lin Yong, Timothy C. Ralph, Sheng-Kai Liao, Juan Yin, Wei- Yue Liu, Wen-Qi Cai, Xuan Han, Hui-Nan Wu, Wei-Yang Wang, Feng-Zhi Li, Meng Yang, Feng-Li Lin, Li Li, Nai-Le Liu, Yu-Ao Chen, Chao-Yang Lu, Yanbei Chen, Jingyun Fan*, Cheng-Zhi Peng*, Jian-Wei Pan*. (2019) Satellite testing of a gravitationally induced quantum decoherence model. Science. (Doi: 10.1126/science. aay5820) 